\title{
Sequencing of five poultry strains elucidates phylogenetic relationships and divergence in virulence genes in Morganella morganii
}

Nicola Palmieri, Claudia Hess, Michael Hess and Merima Alispahic ${ }^{*}$ (]

\begin{abstract}
Background: M. morganii is a bacterium frequently associated with urinary infections in humans. While many human strains are sequenced, only the genomes of few poultry strains are available. Here, we performed a detailed characterization of five highly resistant Morganella morganii strains isolated in association with Escherichia coli from diseased domestic Austrian poultry flocks, namely geese, turkeys and chicken layers. Additionally, we sequenced the genomes of these strains by NGS and analyzed phylogenetic clustering, resistance and virulence genes in the context of host-specificity.

Results: Two strains were identified to be Extended Spectrum Beta Lactamase (ESBL) and one as AmpC betalactamases (AMP-C) phenotype, while two were ESBL negative. By integrating the genome sequences of these five poultry strains with all the available M. morganii genomes, we constructed a phylogenetic tree that clearly separates the Morganella genus into two clusters (M1 and M2), which approximately reflect the proposed subspecies classification (morganii and sibonii). Additionally, we found no association between phylogenetic structure and host, suggesting interspecies transmission. All five poultry strains contained genes for resistance to aminocoumarins, betalactams, colistin, elfamycins, fluoroquinolones, phenicol, rifampin and tetracycline. A comparative genomics analysis of virulence genes showed acquisition of novel virulence genes involved in secretion system and adherence in cluster M2. We showed that some of these genes were acquired by horizontal gene transfer from closely related Morganellaceae species and propose that novel virulence genes could be responsible for expansion of tissue tropism in M. morganii. Finally, we detected variability in copy number and high sequence divergence in toxin genes and provided evidence for positive selection in insecticidal toxins genes, likely reflecting host-related adaptations.
\end{abstract}

Conclusions: In summary, this study describes i) the first isolation and characterization of M. morganii from goose and turkey, ii) a large-scale genetic analysis of $M$. morganii and an attempt to generate a global picture of the $M$. morganii intraspecific phylogenetic structure.

Keywords: Morganella morganii, Poultry, NGS data, MALDI-TOF MS, Antimicrobial resistance, Virulence genes, Phylogeny

\footnotetext{
* Correspondence: merima.alispahic@vetmeduni.ac.at

Clinic for Poultry and Fish Medicine, Department for Farm Animals and Veterinary Public Health, University of Veterinary Medicine, Veterinärplatz 1, 1210 Vienna, Austria
}

(c) The Author(s). 2020 Open Access This article is licensed under a Creative Commons Attribution 4.0 International License, which permits use, sharing, adaptation, distribution and reproduction in any medium or format, as long as you give appropriate credit to the original author(s) and the source, provide a link to the Creative Commons licence, and indicate if changes were made. The images or other third party material in this article are included in the article's Creative Commons licence, unless indicated otherwise in a credit line to the material. If material is not included in the article's Creative Commons licence and your intended use is not permitted by statutory regulation or exceeds the permitted use, you will need to obtain permission directly from the copyright holder. To view a copy of this licence, visit http://creativecommons.org/licenses/by/4.0/ The Creative Commons Public Domain Dedication waiver (http://creativecommons.org/publicdomain/zero/1.0/) applies to the data made available in this article, unless otherwise stated in a credit line to the data. 


\section{Background}

Morganella morganii is a facultative anaerobic Gramnegative enteric rod-shaped bacterium that used to belong to the Enterobacteriaceae family [1]. Recently, Morganella was suggested to be a type genus of a novel Morganellaceae family that consists of the following eight genera: Arsenophonus, Cosenzaea, Moellerella, Morganella, Photorhabdus, Proteus, Providencia, and Xenorhabdus [2]. M. morganii is reported as an opportunistic pathogen in humans and various other animal species [3]. In reported human cases the disease spectrum associated with $M$. morganii infections is broad and the mortality following an infection remains high [3]. In humans it has also been linked with animal bite wound infections, which underlines that $M$. morganii causes zoonotic infectious diseases [3]. However, the bacterium is scarcely reported in poultry: in Nigeria and China, M. morganii was isolated from dead broilers and its pathogenicity confirmed by infecting day-old chicks $[4,5]$. Furthermore, $M$. morganii was isolated from chicken carcasses in US retail raw meat [6] and from a 13-day old broiler from a farm in Portugal [7]. Several factors can affect the progression and severity of an infection with antibiotic resistance as a main factor in both human and veterinary medicine. The World Health Organization has recently published a global priority list of antibiotic-resistant bacteria; "Priority 1: CRITICAL" ranking include extended spectrum $\beta$-lactamase (ESBL) producing and carbapenemase producing $M$. morganii (WHO 2019). M. morganii has a natural resistance to beta-lactam antibiotics (e.g. ampicillins, amoxicillin, most of the 1st and 2nd generation cephalosporins) and colistin [3]. In addition, the presence of pathogenicity determinants is essential to the success of $M$. morganii in any environment. Virulence genes cluster such as urease, flagellar and haemolysin were all found in M. morganii characterizing its potential as a pathogen and the ability to colonise different hosts [7-10]. In other Enterobacteriaceae, association between host and phylogenetic structure was observed in E. coli [11] but not in Salmonella enteritidis [12], neither in Providencia species [13], while the situation for $M$. morganii is presently unknown. A total of 47 complete genome sequences from $M$. morganii strains are currently available from NCBI database mainly isolated from humans, but also one from a broiler, salamander, dolphin, plants and few where the host is unknown. To date, a detailed genome analysis has been carried out for only few of these strains (KT, F675, INSRALV892a, MM 1, MM 4, and MM 190) [7-10] and detailed phylogeny is lacking. These studies reported peculiar strainspecific innovations such as: i) acquisition of resistance genes from Acinetobacter spp. in the F675 strain [9], ii) introduction of toxins (hlyA) through lateral transfer from phages in the MM1, MM4 and MM190 strains [10], and iii) plasmid-mediated acquisition of quinolone resistance in the INSRALV892a strain [7].
Here, we describe five poultry strains of $M$. morganii isolated for the first time from geese, a fattening turkey and a layer chicken parent flock. We characterized their resistance profile phenotypically and analyzed resistance genes, mobile elements and virulence genes through genome sequencing by Illumina NGS. By combining the genomic data of these poultry strains with all the available genomes we constructed a large-scale phylogeny of $M$. morganii, which we used to test the hypothesis of association between host and phylogeny and to highlight phenomena of lineage-specific divergence in virulence genes.

\section{Results}

\section{Necropsy, bacteria isolation and identification}

General fibrinous serositis was observed in dead birds from flocks $A$ and $B$. In case of flock $C$, fibronecrotic typhlitis was the main gross pathological lesion found, a similar observation as in the 13-day old broiler from Portugal [7]. Omphalitis was diagnosed in turkey poults from flock D, while the chicken from a layer parent flock E did lack signs of bacterial infections but had a fracture of the leg. Escherichia coli was isolated from all cases investigated as reported by [7] who detected the same bacterium with $M$. morganii in liver and spleen of a broiler. Riemerella anatipestifer was detected in some organ samples from birds of flock B and Clostridium perfringens was found in association with a fibronecrotic typhlitis. When investigating the $E$. coli isolates for antibiotic resistance by disc diffusion method, smaller colonies were observed within the inhibition zone of ampicillin, amoxicillin and colistin, pointing towards a co-infection of E. coli and M. morganii. MALDI-TOF MS identified all strains to be $M$. morganii species, of which the strain PA17/10312 was classified as subspecies sibonii and the other four strains as subspecies morganii with a $\log$ score value above 2.5 (Table 1). On COS agar $M$. morganii could not be differentiated from $E$. coli or Salmonella spp., as both grow in round shape and appear as greyish smooth colonies. But on McConkey and Coliform agar, colonies were colourless and white (this could be due to the media), respectively, resembling those from Salmonella spp. Thus, morphological similarities to E. coli and Salmonella might have an impact on identification of $M$. morganii in routine diagnostics.

\section{Antibiotic susceptibility testing}

All isolates were found to be multidrug resistant (resistance to more than three antimicrobial substances) by disc diffusion method with resistance patterns ranging from five to ten antibiotics (Table 2). All were resistant to amoxicillin, ampicillin, and colistin, but also to tilmicosin and tylosin. The combination of trimethoprim/ sulfamethoxazole was the only antibiotic to which all five 
Table 1 Details on origin and identification results of M. morganii isolates

\begin{tabular}{llllll}
\hline Flock & Strain Designation & Bird species & Age & Organ & Bacterial isolates \\
\hline A & PA17/10312 & Goose & 5 weeks & brain & Morganella morganii subsp. sibonii, Escherichia coli \\
B & PA18/15564 & Goose & 10 weeks & heart & $\begin{array}{l}\text { Morganella morganii subsp. morganii, Escherichia } \\
\text { coli, Riemerella anatipestifer }\end{array}$ \\
C & PA18/16407 & Goose & 40 weeks & heart, liver, intestine, lung & $\begin{array}{l}\text { Morganella morganii subsp. morganii, Escherichia } \\
\text { coli, Clostridium perfringens }\end{array}$ \\
D & PA18/25921 & Turkey & 1 day & heart & Morganella morganii subsp. morganii, Escherichia coli \\
E & PA19/9695 & Layer parent stock & 32 weeks & heart & Morganella morganii subsp. morganii, Escherichia coli \\
\hline
\end{tabular}

strains were susceptible. Resistance to $\beta$-lactam antibiotics in Morganella species is usually mediated by the presence of chromosomally encoded $\beta$-lactamases belonging to the AmpC $\beta$-lactamase family [14]. These $\beta$-lactamases are typically induced in the presence of $\beta$ lactam antibiotics. The strain PA17/10312 (flock A) was confirmed to be an AMP-C phenotype. In contrast to ESBLs, AmpC hydrolyses broad and extended-spectrum cephalosporins (cephamycins as well as to oxyimino- $\beta$ lactams) but are not inhibited by $\beta$-lactamase inhibitors such as clavulanic acid [15]. The strains PA18/15564 (flock B) and PA18/25921 (flock D) were confirmed to be ESBL phenotype and possible productors of type $\mathrm{D}$ carbapenemases or impermeability/porin loss, whereas the strains PA18/16407 (flock C) and strain PA19/9695 (flock E) were ESBL negative.

\section{Sequencing and annotation of five poultry strains}

The genome sequences of the five poultry strains displayed substantial variation in length and number of genes (Table 3), with the PA17/10312 being longer and having more genes compared to the other strains. To evaluate whether these differences might be due to missing annotations, we computed genome completeness using the program CheckM [17] for each assembly and obtained values of $100 \%$ for all five strains, implying that the observed variation in gene content cannot be attributed to uneven coverage of the assembled strains. Genome size and gene content were also comparable to the assemblies of other published strains [7-10]. Additionally, we observed variations in genome structure among the five strains (Fig. 1a): PA18/15564 and PA18/16407 showed high colinearity compared to the closest reference strain (Additional file 2: Supplementary Figure 1A), while PA17/10312 displayed more genomic rearrangements (Additional file 2: Supplementary Figure 1B). Finally, the genome of the five strains was aligned to the reference strain $\mathrm{KT}$, for which the most comprehensive annotation is available [8]. A multiple alignment in circular form is displayed in Fig. 1b and shows a high level of conservation among the five poultry strains, with few shared gaps, corresponding to the rRNA ribosomal gene clusters, which are notoriously difficult to assemble due to their repetitive nature.

\section{Phylogenetic analysis}

To test the hypothesis of a phylogenetic clustering based on the host in M. morganii, a phylogenetic tree was constructed including the five sequenced poultry isolates together with all available complete genomes from NCBI, encompassing a total of 52 strains. The genome-based k-mer tree displayed two distinct subtrees (M1 and M2),

Table 2 Antibiotic susceptibility testing by disc diffusion method $-\mathrm{R}=$ Resistant, $\mathrm{S}=$ Susceptible, $\mathrm{I}=$ Intermediate

\begin{tabular}{|c|c|c|c|c|c|}
\hline Antibiotics $(\mu \mathrm{g})$ & PA17/10312 & PA18/15564 & PA18/16407 & PA18/25921 & PA19/9695 \\
\hline Amoxicillin (10) & $R$ & $R$ & $R$ & $R$ & $\mathrm{R}$ \\
\hline Ampicillin (10) & $\mathrm{R}$ & R & $\mathrm{R}$ & $\mathrm{R}$ & $\mathrm{R}$ \\
\hline Colistin (10) & $\mathrm{R}$ & $\mathrm{R}$ & $\mathrm{R}$ & $\mathrm{R}$ & $\mathrm{R}$ \\
\hline Doxycycline (30) & $\mathrm{R}$ & I & $R$ & $\mathrm{R}$ & S \\
\hline Enrofloxacin (5) & S & । & $\mathrm{R}$ & $\mathrm{R}$ & $\mathrm{R}$ \\
\hline Neomycin (30) & । & । & । & S & S \\
\hline Oxalic Acid (2) & S & S & S & $\mathrm{R}$ & $\mathrm{R}$ \\
\hline Spectinomycin (100) & S & S & S & $\mathrm{R}$ & S \\
\hline Tetracycline (30) & । & 1 & $\mathrm{R}$ & $\mathrm{R}$ & S \\
\hline Tilmicosin (15) & $\mathrm{R}$ & $\mathrm{R}$ & $\mathrm{R}$ & R & $\mathrm{R}$ \\
\hline Trimethoprim/Sulfamethoxazole (25) & S & s & S & S & S \\
\hline Tylosin (150) & $\mathrm{R}$ & $\mathrm{R}$ & $\mathrm{R}$ & R & $\mathrm{R}$ \\
\hline
\end{tabular}


Table 3 Genomic features of the five sequenced poultry strains

\begin{tabular}{|c|c|c|c|c|c|}
\hline & PA17/10312 & PA18/15564 & PA18/16407 & PA18/25921 & PA19/9695 \\
\hline Number of contigs & 78 & 59 & 47 & 548 & 667 \\
\hline Total length (bp) & $4,085,866$ & $3,675,879$ & $3,702,167$ & $4,066,432$ & $3,988,823$ \\
\hline N50 (bp) & 244,109 & 403,330 & 989,625 & 403,126 & 49,931 \\
\hline GC content & $50.2 \%$ & $51.2 \%$ & $51.2 \%$ & $51.0 \%$ & $51.6 \%$ \\
\hline Genes & 3840 & 3452 & 3490 & 3812 & 3756 \\
\hline Coding genes & 3784 & 3393 & 3431 & 3718 & 3671 \\
\hline Genome completeness & $100 \%$ & $100 \%$ & $100 \%$ & $100 \%$ & $100 \%$ \\
\hline Contamination score $(\%)^{a}$ & 0.00 & 0.54 & 0.00 & 1.92 & 2.75 \\
\hline Strain heterogeneity ${ }^{\mathrm{b}}$ & 0.00 & 0.00 & 0.00 & 37.50 & 50.00 \\
\hline
\end{tabular}

a,b For a detailed description of these metrics, see the CheckM paper [16]

${ }^{b}$ The strain heterogeneity $(\mathrm{SH})$ index indicates the proportion of the contamination that appears to be from the same or similar strains and is a number between 0 and 100

with no apparent clustering according to the host they were isolated from (Fig. 2). The resulting tree places the strains PA18/16407, PA18/15564, PA19/9695, PA18/ 25921 in cluster M1 and the PA17/10312 strain in cluster M2. Historically, M. morganii has been divided into two subspecies: morganii and sibonii, based on the ability to ferment trehalose and other biochemical features [18]. Thus, we hypothesized that clusters M1 and M2 simply reflect the known subspecies classification. To test this idea, the presence of the trehalose operon (treR, tre $B$ and $\operatorname{tre} P$ ) in all the strains was investigated. By this approach, the subspecies sibonii was assigned to

\section{(A)}

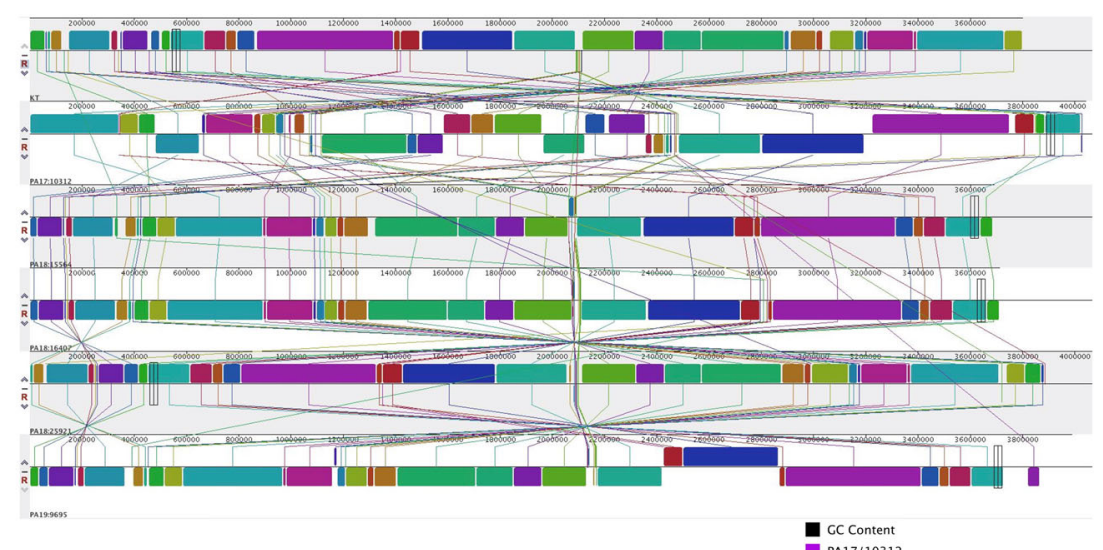

(B)

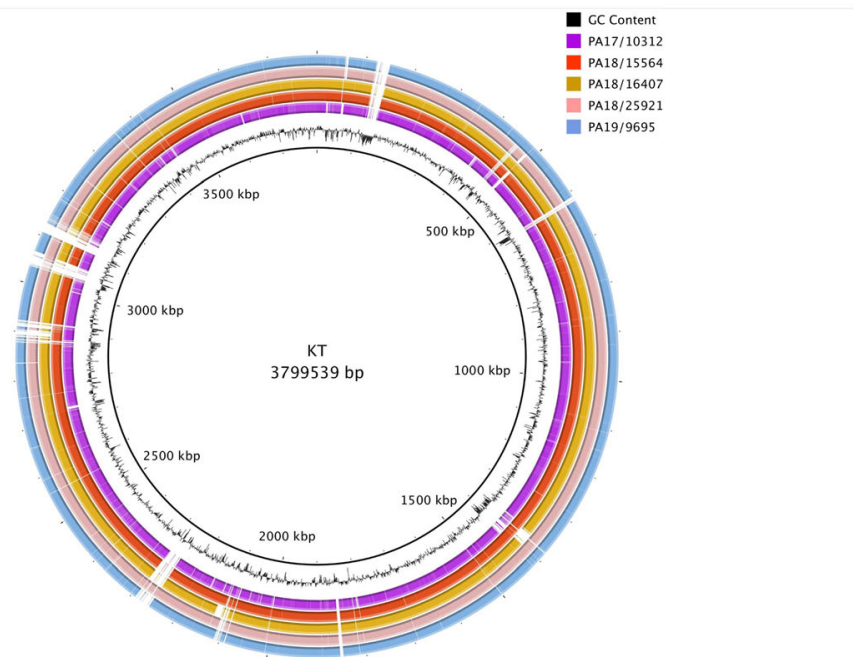

Fig. 1 a Multiple genome alignment of the five sequenced poultry strains and the KT reference strain $\mathbf{b}$ Circular genomic map constructed using BRIG displaying a multiple genome alignment of the five poultry strains and the KT reference strain 


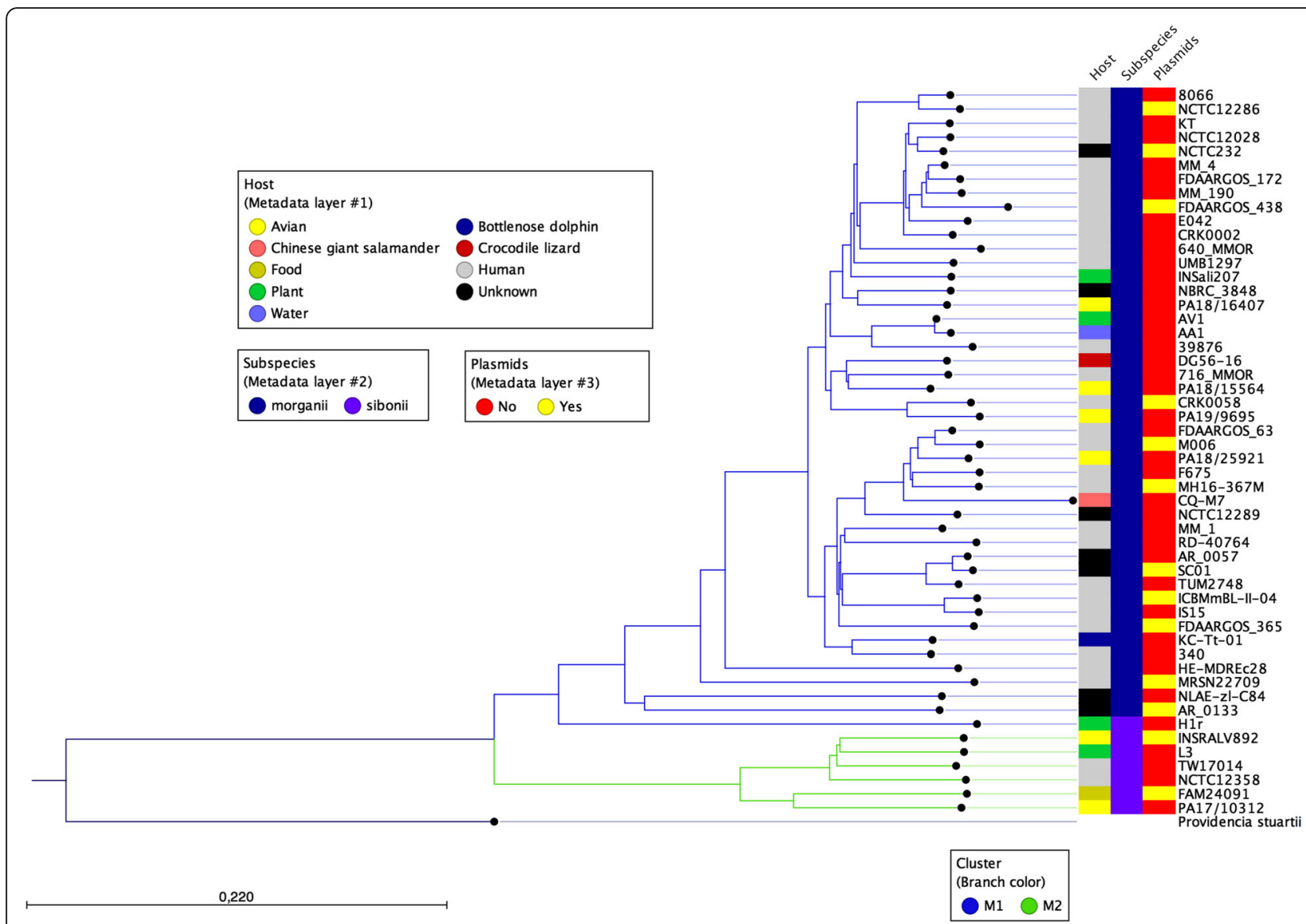

Fig. $2 \mathrm{~K}$-mer tree including the five sequenced poultry strains and 47 complete genomes from NCBI, using Providencia stuartii as an outgroup

the strains in which the trehalose operon was present. Comparing this classification to the observed phylogenetic clustering, we found that the sibonii strains were entirely allocated in cluster M2, except the H1R strain (Fig. 2), confirming that differentiation of subspecies can be mostly performed based on phylogenetic grouping. These results are consistent with the subspecies assignment achieved by MALDI-TOF MS (Table 1). To validate the accuracy of the k-mer tree reconstruction, an additional tree was built using a whole-genome based approach using the program parsnp (Additional file 3: Supplementary Figure 2A). Comparison of both trees showed high similarity in topology with only minor differences: i) the NCTC12358 and TW17014 strains of cluster M2 are swapped in the whole-genome based tree compared to the k-mer tree; ii) the 640_MMOR strain is grouped together with NCTC12286 and 8066 in the whole-genome based tree, while in the $\mathrm{k}$-mer tree constitutes a separate cluster. Since recombination can sometimes distort branch lengths in bacterial wholegenome based phylogenies (i.e. [19]), we performed another tree reconstruction by filtering SNPs located in putative regions of recombination (parsnp parameter $-\mathrm{x}$
YES) (Additional file 3: Supplementary Figure 2B). However, we did not find significant differences in branch length compared to the uncorrected tree (MannWhitney test $-P=0.9914)$. As the differences between the whole-genome based trees and the k-mer tree do not affect the conclusions derived from following analyses, the k-mer tree was used as a guide for the remainder of the analyses.

\section{Detection of resistance genes}

The sequences of all five poultry strains contained genes for resistance to aminocoumarins, beta-lactams, elfamycins, fluoroquinolones, rifampin and tetracycline. Resistance to phenicol was present in all the sequenced poultry strains, except in the chicken strain PA19/9695. Based on BLAST searches against the MEGARes database, the investigation of presence/absence of resistance genes in all 52 strains from Fig. 2 revealed that resistance to certain antibiotics is widespread in Morganella. This includes aminocoumarins, beta-lactams, elfamycins, fluoroquinolones, phenicol, rifampin and tetracyclines (Fig. 3). Other types of resistance show a more uneven distribution along the tree, with multiple cases of strain- 


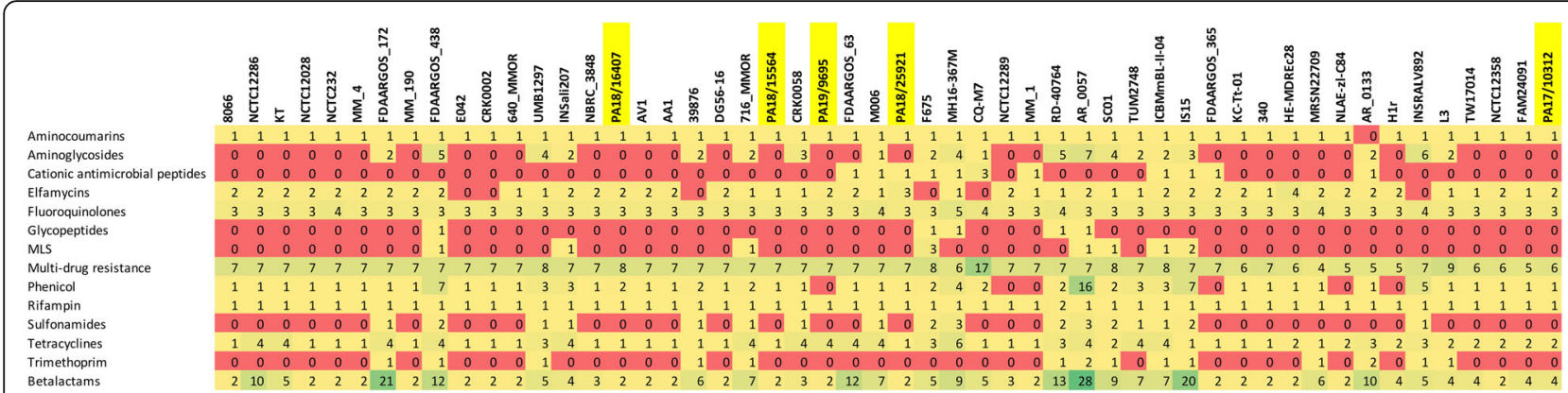

Fig. 3 Phylogenetic distribution of resistance genes in the 52 analyzed M. morganii strains ordered according to the position in the tree from Fig. 2. The five sequenced poultry strains are highlighted in yellow. Every cell contains the number of gene copies involved in a certain type of resistance for each strain. Cells are coloured using conditional formatting to facilitate readability

specific gains, like resistance to glycopeptides, which is present in 5/52 strains with no apparent phylogenetic clustering. This suggests that these genes could have originated by horizontal gene transfer. Additionally, we looked at resistance genes that were absent in the MEGARes database, but that were previously reported for other strains of $M$. morganii, such as genes involved in lipid A biosynthesis $(l p x A, l p x B, \operatorname{lp} x C, \operatorname{lp} x D, \operatorname{lp} x H, \operatorname{lp} x K, \operatorname{lp} x L$, $l p x M, l p x P$ and $l p x T)$, which are responsible for colistin resistance [9]. Such analyses detected lpxABCDHKLMPT in both geese strains, lpxABCDHKLMT in the chicken strain PA19/9695, lpxABCDHKLMT in the turkey strain PA18/25921 and lpxABCDHLMT in the goose strain PA17/10312. An additional copy of the $\operatorname{lpxP}_{\text {gene was }}$ found in PA18/25921 and PA17/10312. Also, the tetB gene responsible for tetracycline resistance was found in all five poultry strains. No association was detected between resistance genes of a certain class and a specific phylogenetic cluster. These results are concordant with the phenotypic tests of antibiotic resistance (Table 2).

\section{Detection of known virulence genes}

In an initial approach, the sequences from the poultry strains were screened for known virulence genes based on the comparison with the KT strain, for which an exhaustive list of virulence genes is available. However, the gene-id based detection of genes from the KT strain was unfeasible due to incongruences between gene-ids from the KT strain and the ones deposited in the NCBI gene database, thus the 4-letter gene name was used to retrieve the genes of interest, resulting in a partial set of 125 genes for this analysis. Most of the known virulence genes present in this set were found in the sequences of the five poultry strains (Fig. 4). This not only indicates that virulence genes are very conserved, but also suggests that the annotations of the five poultry strains is highly complete. As this gene set did not include all known virulence genes, we additionally looked for the presence of specific classes of virulence genes of importance. Hemolysins are essential for pore-formation during invasion of host cells [20] and encoded by the hlyCABD operon. This operon was present and intact only in the chicken strain PA18/25921, while absent in PA18/16407, PA18/15564 and PA19/9695. In the sibonii strain PA17/10312, only $h l y C$ and $h l y D$ were present and distributed on different contigs. Ureases are also typically organized into the ureABCFGD operon and contribute to formation of urinary stones [21]. This operon was present and intact in all five strains. Finally, the capsule synthesis regulation genes $r c s B, r c s C, r c s D$ and $r c s F$ involved in host immune response [22] were also detected in all five strains, with an additional copy of $r c s B$ in PA18/25921. Interestingly, virulence genes in the category "toxins" displayed high variation in copy numbers among the five strains (Fig. 4). Manual scanning of the alignments also showed substantial variation in coding sequences exemplarily shown for the RtxA toxin in Additional file 4: Supplementary Figure 3. For these reasons, a positive selection scan using PAML (see Materials and methods) was performed based on the hypothesis that toxins might evolve under positive selection in $M$. morganii. For this analysis, only toxins present in all five strains were selected (Fig. 4) and it was found that four out of nine toxins $(t c a C / t c d B 2$, $x p t A 1, x p t B 1, x p t C 1)$ showed signs of positive selection using branch models by selecting either cluster M1 or M2 as the target branch (Table 4). Notably, the insecticidal proteins XptA1B1C1 had the strongest signal of positive selection.

\section{Detection of novel virulence genes}

To get an overview of the variation in individual virulence genes across the strains, the number of virulence genes of different functional categories in each strain was plotted and sorted according to the phylogenetic position on the tree (Fig. 5). An increase in number of genes in strains of cluster M2 compared to the cluster M1 in the virulence categories adhesion, secretion 

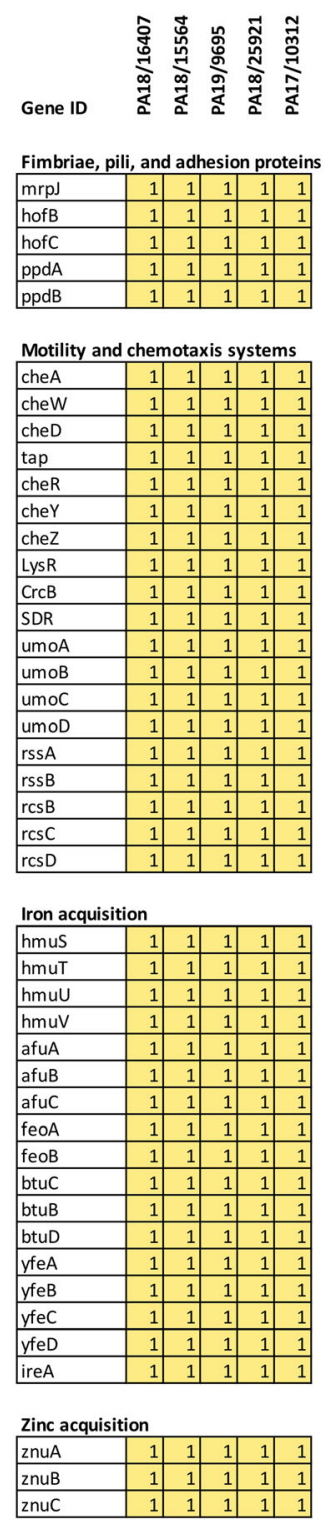

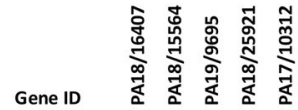

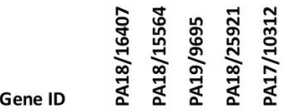

Type III secretion system
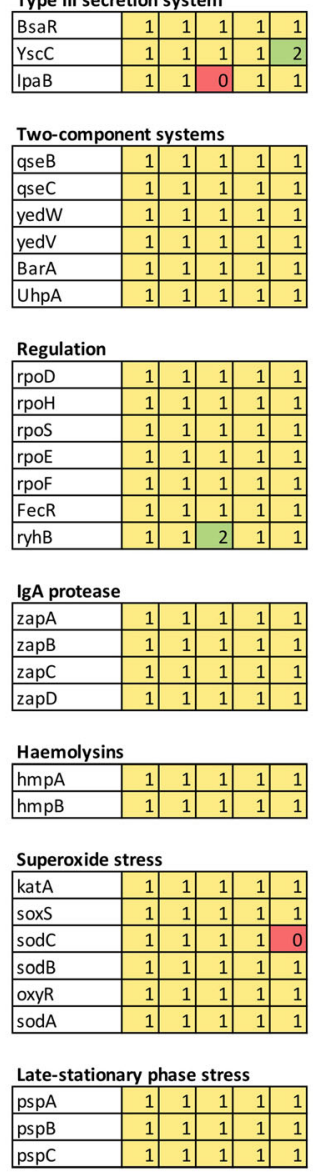

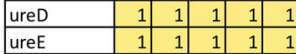

\section{Urease hydrolysis and putrescine production}

\begin{tabular}{|l|l|l|l|l|l|}
\hline ureA & 1 & 1 & 1 & 1 & 1 \\
\hline
\end{tabular}

\begin{tabular}{|l|l|l|l|l|l|}
\hline ureB & 1 & 1 & 1 & 1 & 1 \\
\hline ureC & 1 & 1 & 1 & 1 & 1 \\
\hline ureD
\end{tabular}

\begin{tabular}{|l|l|l|l|l|l|}
\hline ureC & 1 & 1 & 1 & 1 & 1 \\
\hline ureD & 1 & 1 & 1 & 1 & 1 \\
\hline
\end{tabular}

\begin{tabular}{|l|r|r|r|r|r|}
\hline ureF & 1 & 1 & 1 & 1 & 1 \\
\hline & 1 & 1 & 1 & 1 & 1 \\
\hline
\end{tabular}

\begin{tabular}{|l|l|l|l|l|l|}
\hline ureG & 1 & 1 & 1 & 1 & 1 \\
\hline
\end{tabular}

\begin{tabular}{|l|l|l|l|l|l|}
\hline ureH & 1 & 1 & 1 & 1 & 1 \\
\hline
\end{tabular}

\begin{tabular}{|l|l|l|l|l|l|}
\hline speF & 2 & 2 & 2 & 2 & 2 \\
\hline potE
\end{tabular}

\begin{tabular}{|l|r|r|r|r|r|}
\hline potE & 1 & 1 & 1 & 1 & 1 \\
\hline carA & 1 & 1 & 1 & 1 & 1 \\
\hline carB
\end{tabular}

\begin{tabular}{|l|r|r|r|r|r|}
\hline carA & 1 & 1 & 1 & 1 & 1 \\
\hline carB & 1 & 1 & 1 & 1 & 1 \\
\hline
\end{tabular}

\begin{tabular}{|l|r|r|r|r|r|}
\hline carB & 1 & 1 & 1 & 1 & 1 \\
\hline $\operatorname{argl}$ & 1 & 1 & 1 & 1 & 1 \\
\hline $\arg$
\end{tabular}

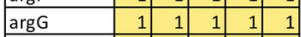

\begin{tabular}{l|l|l|l|l|l|}
\hline $\operatorname{argH}$ & 1 & 1 & 1 & 1 & 1 \\
\hline $5 p e$
\end{tabular}

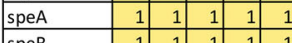
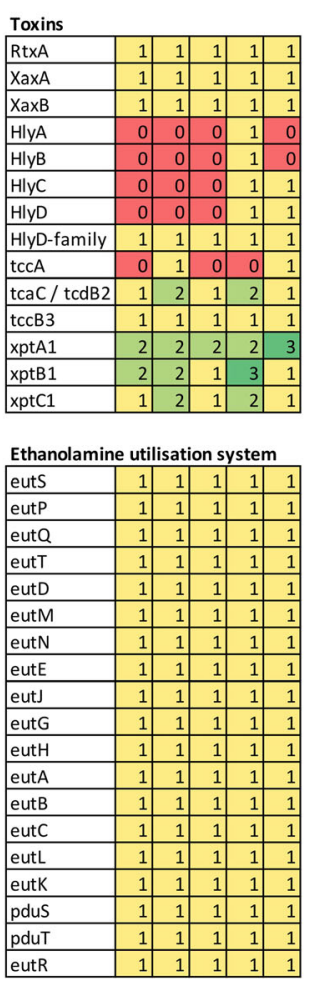

Fig. 4 Comparison of known virulence genes in the five sequenced poultry strains using the KT strain as reference

system and hypothetical was observed (Fig. 5). As the strain PA17/10312 is also located in cluster M2, these results might explain the higher number of genes in the PA17/10312 strain compared to the other strains. Novel virulence genes were defined as those specific to cluster M2: a total of 39 novel virulence genes were found in this way (Table 5). To validate specificity to cluster M2, these genes were BLASTed to two outgroup species: Providencia stuartti and Proteus mirabilis, and only genes with no hits to both species were retained. This resulted in a final set of 26 novel virulence genes.
Regarding phylogenetic distribution, 10/26 genes were shared by all strains in cluster M2, consistent with ancestral gain in cluster M2 and 12/26 genes were specific to a single strain, likely due to strain-specific horizontal gene transfer events. To find evidence for horizontal gene transfer the novel virulence genes were BLASTed against the whole bacterial protein database. Then, using the program Alienness [23], evidence for horizontal gene transfer (HGT) was detected in 6/26 (23\%) of the novel virulence genes ( $a f a B, e s c U$, escV, SG1030, stx2A, ycbV), having an Alien Index (AI) $>30$ (Additional file 5: 
Table 4 List of toxins and associated $p$-values for the positiveselection test using cluster M2 or M1 as target branch. Significant genes are highlighted in grey

\begin{tabular}{lll}
\hline Toxin & $\boldsymbol{P}$-value M2 & $\boldsymbol{P}$-value M1 \\
\hline RtxA & 1.0000 & 1.0000 \\
XaxA & 0.9980 & 0.6633 \\
XaxB & 0.9546 & 1.0000 \\
HlyD-family & 1.0000 & 1.0000 \\
tcaC / tcdB2 & 0.0200 & 0.0200 \\
tccB3 & 1.0000 & 1.0000 \\
XptA1 & 0.0003 & 0.0006 \\
XptB1 & 0.0010 & 0.0010 \\
XptC1 & 0.0036 & 0.0009 \\
\hline
\end{tabular}

Supplementary Table 2). These genes were assigned to the different donors of the class Morganellaceae (Additional file 5: Supplementary Table 2).

\section{Prediction of pathogenic potential for humans}

Using PathogenFinder 1.1 [24] the pathogenic potential of the five poultry strains for humans was estimated. The approach used by this tool is based on the presence of group of genes that are frequently associated with human pathogenic bacteria. Results showed that the PA17/10312 was predicted to be pathogenic in humans
(Probability $=0.64$ ), while the four other strains were classified as non-pathogenic in humans (Probability= 0.57 for PA18/15564, 0.52 for PA18/16407, 0.57 for PA18/25921 and 0.54 for PA19/9695). These results are consistent with the higher number of virulence factors found in PA17/10312 compared to the other strains. It remains to be determined in an animal trial whether these findings also reflect the host-specific pathogenicity.

\section{Annotation of mobile elements}

Mobile elements such as plasmids, prophages and integrons are often responsible for transfer of resistance genes in bacteria $[25,26]$. No evidence for plasmids was found in any of the five poultry strains using two independent approaches (see Materials and methods). As plasmid information is only available for some of the published strains (i.e. [7, 8, 27-29]), we screened all the strains of Fig. 2 using PlasmidFinder [16] for the presence of plasmids and results are shown in Fig. 2 (Metadata layer 3). A total of four active prophages were detected in PA17/10312, three in PA18/15564, two in PA18/16407, five in PA18/25921 and one in PA19/9695 (Additional file 6: Supplementary Table 3), none of them showing association with resistance genes. Two integrons were found in the PA19/9695 strain and one integron was found in the PA17/10312 strain, also not associated with resistance genes. Pathogenicity islands are another class of

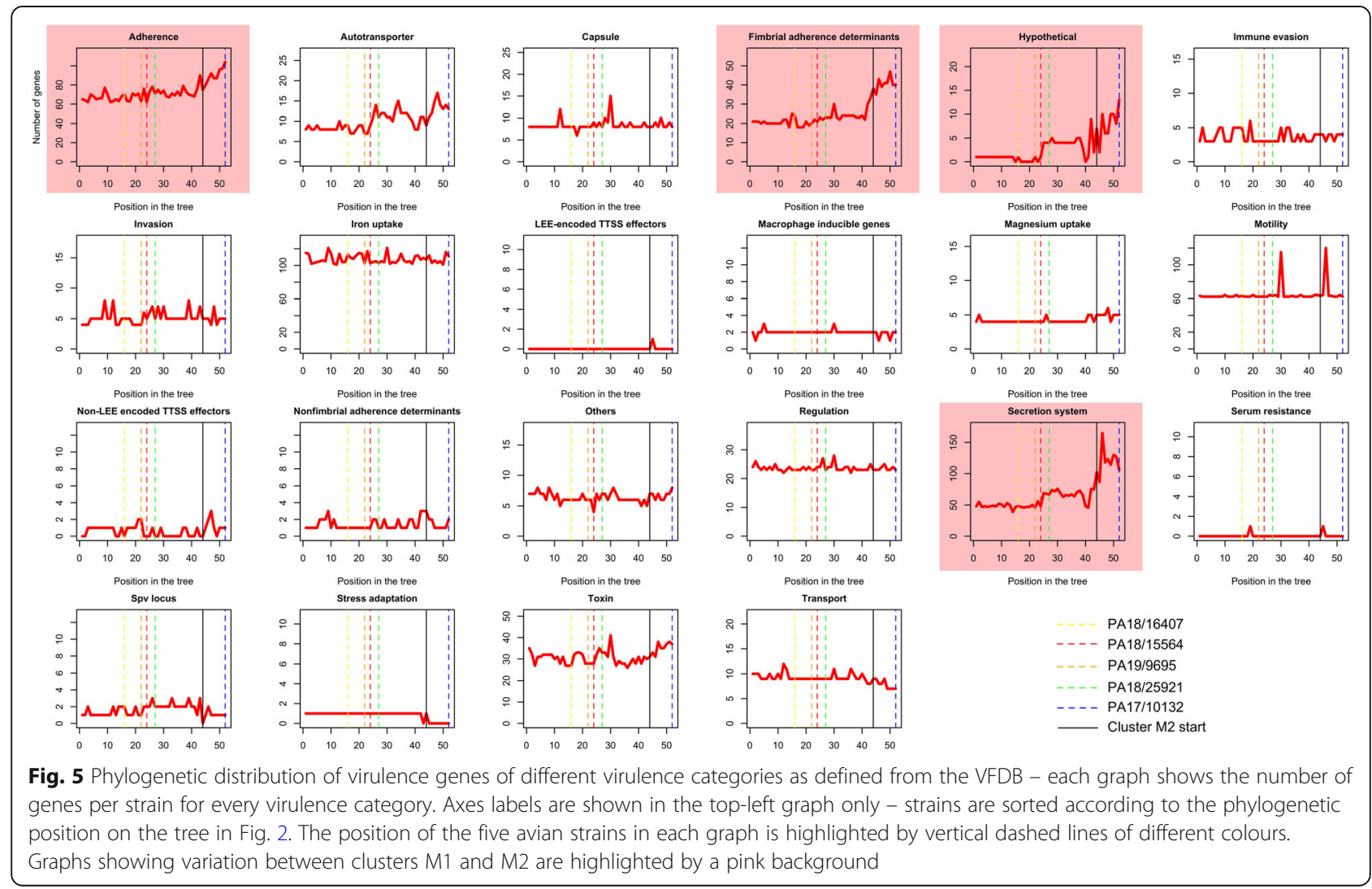


Table $\mathbf{5}$ List of novel virulence genes specific to the M2 cluster

\begin{tabular}{ll}
\hline Category & Genes \\
\hline Adherence & $\begin{array}{l}a f a A B, \text { eae, fdeC, pagN, papADF, pixACH, sfaG, sfpAC, } \\
\text { stiC, stkB, ycbV }\end{array}$ \\
Secretion system & $\begin{array}{l}\text { aatA, EC55989_3333, ECP_0235, ECVR50_0257, } \\
\text { escNSTUV, espD, LF82_024, mxiH, O3M_04340, pipB, } \\
\text { prgl, sipB }\end{array}$ \\
Hypothetical & SG1030 \\
Toxins & IbN, stx2A, stXA \\
Iron uptake & fyuA \\
Others & STM0570 \\
\hline
\end{tabular}

mobile elements, which are linked with virulence genes in a large number of bacteria [30]. A total of 18 pathogenicity islands were detected in PA17/10312, 16 in PA18/15564, 21 in PA18/16407, 22 in PA18/25921 and 23 in PA19/9695 (Additional file 7: Supplementary Table 4, Additional file 8: Supplementary Figure 4), associated, respectively, with 25, 9, 23, 16 and 24 virulence genes. The most abundant virulence categories associated with these genes were: "adherence" for 20/25 genes in PA17/10312, "adherence" for 5/9 genes in PA18/15564 and "iron uptake" for $11 / 23$ genes in PA18/16407, "toxins" for 5/16 genes in PA18/25921 and "adherence" for $11 / 24$ in PA19/9695. A striking feature of pathogenicity islands is that they are located in regions of low genomic conservation, (Additional file 8 - Supplementary Figure 4), suggesting that they were acquired by phenomena of lateral gene transfer.

\section{Identification of strain-specific metabolic pathways}

To study metabolic pathways, the proteomes of the five poultry strains were submitted to BlastKOALA [31], which provides a global overview of metabolic pathways displayed as a metabolic network. This program also allows an easy comparison of metabolic networks among different organisms or strains. All metabolic pathways were entirely conserved among the five poultry strains. In addition, two novel pathways specific to the PA17/ 10312 strain were discovered (Additional file 9: Supplementary Figure 5): sucrose catabolism and biosynthesis of betaine from choline. Betaine is an osmoprotectant that allows organisms to grow in high-osmolarity environments [32]. Some bacteria can synthesize betaine de novo from intermediates of central metabolism whereas others can synthesize it only from exogenously supplied choline [33]. Screening all the $52 \mathrm{M}$. morganii strains included in Fig. 2 revealed that only the FAM24091 strain (isolated from Switzerland cheese), which is the closest strain to PA17/10312, possess genes for betaine biosynthesis (BetA and BetB). No evidence for horizontal gene transfer was found, based on the absence of proteins from closely related species which would cluster together with BetA and BetB from PA17/10312 based upon BLAST analyses. Whether the determined pathways are beneficial for the bacteria in vitro and in vivo remains speculative for the moment and needs to be determined in additional studies.

\section{Discussion}

In the last years, many studies isolated and sequenced Morganella morganii strains from human [8-10, 27-29] while the genomic characterization for other species is scarce and include a strain from chicken [7], cattle [34], together with strains from various hosts that were deposited in NCBI but not described in details (see Fig. 2). Here, we expanded the collection of avian M. morganii by sequencing five novel strains from goose, turkey and chicken. Understanding the relationship between host and phylogeny is useful to study population structure and uncover the origin and evolution of novel strains, in order to understand the emergence of potential outbreaks. Overall, the phylogenetic analysis showed no association between host and a particular phylogenetic cluster, consistent with previous studies also reporting lack of association with host in M. morganii [27, 34]. This characterizes $M$. morganii as a species with no specific population structure. Regarding the subspecies classification, we found that four of the avian strains (PA18/15564, PA18/16407, PA18/25921 and PA19/ 9695) were assigned as subsp. morganii, while the strain PA17/10312 was assigned to subsp. sibonii based on phylogenetic clustering. When looking at the tree including all the available strains, we found that one strain (H1r) classified as sibonii based on the presence of the trehalose genes was assigned to cluster M1, implying that the characterization of subspecies in M. morganii purely based on the presence of the trehalose fermentation genes or the phylogenetic clustering pattern alone can sometimes be imprecise. Antibiotic resistance is another important feature of clinical relevance, in this regard it was previously shown that another avian $M$. morganii strain was multidrug resistant [7], raising concerns for the control of infections in poultry production. In this study, we found that all five strains were resistant to aminocoumarins, beta-lactams, elfamycins, fluoroquinolones, rifampin and tetracycline. Importantly, these classes of resistance were also widespread in all sequenced strains from Fig. 2. The antibiotic resistance results are in agreement with earlier data describing ESBL producers as multiple drug resistant [35], also the classes of resistance determined computationally were consistent with a previously compiled review of resistance genes [3]. Additionally, the genomic characterization allowed us to compare presence of specific virulence genes associated with certain strains or phylogenetic 
clusters. Within the essential virulence genes, ureases and capsule synthesis regulation genes were very conserved in all five strains, while haemolysins showed more variability. Variation in hemolysin composition was also observed in three recently sequenced human strains [10]. A general pattern emerged by looking at the distribution of virulence genes among all sequenced strains, namely we observed that strains of the subspecies sibonii had on average more virulence genes compared to the subspecies morganii and were particularly enriched in virulence genes involved in secretion and adhesion. These results were also corroborated by the prediction that sibonii strains were found to be potentially pathogenic for human. Through comparative genomics analysis, we pinpointed 26 virulence genes as specific to the subspecies sibonii and showed that some of them might have originated by horizontal gene transfer from closely related Morganellaceae species. Among these genes, $a f a A B$ [36], pixC [37], sfaG and fyuA [38] are known to be associated with adhesion and colonization of urinary tract in E. coli, while the intimin $f d e C$ [39], the Shiga toxins stx2A/stxA [40], escN [41], espD [42] are connected with enteropathogenic strains of $E$. coli. This suggests that some $M$. morganii sibonii strains might have acquired the ability to invade the enteric tract. Another important classes of virulence genes are toxins, for which we showed evidence for positive selection, especially high in the insecticidal toxins XptA1B1C1. While the precise function of these toxins in $M$. morganii is unknown, it was shown in Xenorhabdus spp. and Photorhabdus luminescens that they facilitate the killing of the insect host via nematodes living in symbiosis with these bacteria [43]. Thus, positive selection in insecticidal toxins might reflect an evolutionary arms race among species.

\section{Conclusion}

In the actual study, five highly resistant Morganella morganii strains from diseased poultry flocks were isolated in association with $E$. coli. The strains were identified by MALDI-TOF MS down to subspecies level. Micronaut characterization revealed two strains to be ESBL and one strain to be AMP-C phenotypes. To gather more detailed information about their association with the host, resistance profile, virulence and zoonotic potential, the genomes of the five strains were sequenced and compared with all available complete $M$. morganii genomes. The phylogenetic structure of $M$. morganii separates all strains into two main phylogenetic clusters (M1 and M2) that approximately reflect the subdivision of the Morganella clade into the known subspecies morganii and sibonii, with no association between phylogenetic structure and host. Additionally, we showed that cluster M2 diverged through acquisition of novel virulence genes involved in adherence and secretion and that some of these genes might have originated by horizontal gene transfer from closely related Morganellaceae species. Furthermore, high sequence divergence in toxins was detected and evidence for positive selection for some of these genes was provided. Finally, we reported detailed resistance profiles for all so far sequenced $M$. morganii strains complementing the phenotypic resistance profile of the five poultry strains. Altogether, this study represents the first large-scale comparative study in $M$. morganii and represents a useful resource to aid future clinical management of infections and for advancing the field of comparative genomics of Morganella. Performing experimental studies will be crucial to further clarify the nature of $M$. morganii as an avian pathogen and its zoonotic potential.

\section{Methods}

\section{Necropsy, bacteria isolation and identification}

During routine diagnostics the Clinic of Poultry and Fish Medicine (Vetmeduni Vienna, Austria) obtained carcasses from three geese flocks (A-C), one-day old turkey (D) and one-layer chicken parent (E) flocks (Table 1) for necropsy and bacteriological investigation. Necropsy and bacteriology were performed in the diagnostic unit of the clinic accredited according to EN ISO/IEC 17025 applying standard operating procedures. For bacteriological investigation, direct smears were taken aseptically from heart, liver, intestine, brain, spleen and lungs. Samples from each organ were cultivated on Columbia (COS) agar containing $5 \%$ sheep blood (BioMerieux, Vienna, Austria), McConkey agar (Bertoni, Vienna, Austria), Chromocult Coliform agar (Merck, Vienna, Austria) and subsequently incubated aerobically at $37^{\circ} \mathrm{C}$ for $24 \mathrm{~h}$. Additionally, intestinal samples were cultivated on Schaedler (SCS) agar containing 5\% sheep blood (BioMerieux, Vienna, Austria) for anaerobic growth (Genbox anaer BioMerieux) incubated at $37^{\circ} \mathrm{C}$ for $24 \mathrm{~h}$ as well as for fungal growth on Sabouraud Gentamicin Chloramphenicol (SGC2) agar (BioMerieux, Vienna, Austria) incubated at $42{ }^{\circ} \mathrm{C}$ for $48 \mathrm{~h}$. All bacterial isolates were identified by Matrix Assisted Laser Desorption/Ionization-Time of Flight Mass Spectrometry (MALDI-TOF MS) (Microflex LT instrument, Bruker Daltonic) against the reference library from Bruker Daltonic version 4.1.80 according to the manufacturer's instructions, with sample preparation performed as previously described [44].

\section{Antibiotic susceptibility testing}

Antibiotic susceptibility testing was performed by disc diffusion method according to the EUCAST guidelines ([45], EUCAST disc diffusion method, version 6.0, 2019. http://www.eucast.org/clinical_breakpoints/). Phenotypic detection of ESBL type A $\beta$-lactamases, AmpC type $\mathrm{C}$ 
cephalosporinases, KPC type A carbapenamases and MBL metallo- $\beta$-lactamases was performed by broth microdilution method using the commercially available MICRONAUT-S ß-Lactamases plateE1-111-040 (MERL IN Diagnostika GmbH, Bornheim-Hersel, Germany). The antimicrobial substances and their concentration are given in Additional file 1: Supplementary Table 1. The analysis was done according the manufacturer's instructions. Results were evaluated with the MCN6 Software Version 6.00 Release 71 (MERLIN Diagnostika GmbH, Bornheim-Hersel, Germany).

\section{Genome sequencing and annotation}

DNA was extracted using QIAamp DNA Mini Kit following the manufacturer's guidelines (Qiagen, Hilden, Germany). The five samples were sent to the Vienna BioCenter Core Facility, where they were sequenced on an Illumina NextSeq machine using a paired-end PCRfree library (150 bp read length). Resulting reads were assembled into contigs using CLC Genomics Workbench 12.0 (https://www.qiagenbioinformatics.com/) by the De Novo Assembly workflow (default parameters) and annotated using PROKKA 1.13 [46]. Plasmids were detected using PlasmidFinder 2.0 [16] and by BLAST searches against a local version of the Enterobacteriaceae plasmid database [47]. Prophages were annotated using the recent tool Prophage Hunter [48], which is also able to differentiate active from inactive prophages. Only active prophages were selected into the final annotation. Integrons were annotated by IntegronFinder 1.5.1 [49]. Pathogenicity islands were identified by IslandViewer 4 [50] using the M. morganii KT strain [8] as a reference.

\section{Phylogenetic trees construction}

For tree construction, the five sequenced assemblies were employed together with all 47 complete $M$. morganii genomes downloaded from NCBI (see Additional file 10 Supplementary Table 5 for a list of GenBank accession IDs) encompassing a total of 52 strains. For each strain, the genome sequence was concatenated to form a single artificial sequence, including plasmids and unscaffolded sequences. A k-mer phylogenetic tree was constructed using CLC Microbial Genomics 12.0 by the Create K-mer Tree workflow (default parameters). Additional phylogenetic trees were constructed based on multiple genome alignment of all the strains with the program parsnp [51], using the KT strain [8] as a reference.

\section{Identification of resistance genes}

The protein sequences of each strain were BLASTed (blastp -evalue 1e-05) to a local version of the MEGARes database [52], which contains more than 4000 handcurated antimicrobial resistance genes. Hits with a query coverage $>40 \%$ were kept for further analyses. Additional screening for resistance genes that were absent in the MEGARes database (lpx genes, tet $B$ ) was performed using the same BLAST-based approach.

\section{Identification of virulence genes}

The protein sequences of virulence genes for the $M$. morganii KT strain [8] (GenBank ID GCA_000286435.2) were retrieved using the 4-letter gene-id from the NCBI Protein database and BLASTed (blastp -evalue 1e-05) to the proteomes of the five sequenced poultry strains. Hits with a query coverage $>40 \%$ were kept and processed. Additional virulence genes were identified by BLASTing the protein sequences of the five strains to a local version of the VFDB (Virulence Factor DataBase) using stringent criteria (blastp -evalue 1e-10, query coverage $>40 \%$ ), in order to get a conservative set of virulence genes. Novel genes were detected in the following way: the proteomes of the 52 strains of M. morganii from Fig. 2, together with the representative NCBI strain of $P$. stuartii and P. mirabilis (as outgroups) were BLASTed to the VFDB with stringent criteria (blastp -evalue 1e-10, query coverage $>40 \%$ ) and a table was generated containing the phyletic patterns of each virulence gene in every strain. Novel genes were then defined as genes-specific to cluster M2 (see Fig. 2). Annotation of functional classes for virulence genes was based on the intra-genera VFs comparison tables from VFDB (http://www.mgc.ac.cn/ VFs/Down/Comparative_tables_from_VFDB.tar.gz).

Gene families were computed by clustering the protein sequences of each strain using the sequence clustering program MMseqs2 [53] with a minimum $80 \%$ similarity threshold.

\section{Positive-selection scans}

Coding sequences for orthologous groups of genes of interest were aligned using PRANK [54]. Positive selection scans were performed with the codeml module from PAML [55] by comparing a model with a fixed $\omega$ for the whole tree (model $=2$, NSsites $=2$, fix_omega $=$ 1) with a model allowing positive selection in the M2 cluster (Fig. 2) $($ model $=2$, NSsites $=2$, fix_omega $=0$ ) or in the M1 cluster (model $=2$, NSsites $=2$, fix omega $=0$ ). $P$-values were calculated by standard loglikelihood ratio tests.

\section{Identification of strain-specific metabolic pathways}

Protein sequences were submitted to BlastKOALA [31] to reconstruct the metabolic network of each strain. The metabolic networks of the different strains were compared through the KEGG Pathway interface from the KEGG database [56] in order to discover strain-specific pathways. 


\section{Supplementary information}

Supplementary information accompanies this paper at https://doi.org/10. 1186/s12864-020-07001-2.

Additional file 1: Table S1. MICRONAUT antimicrobial substances and concentrations.

Additional file 2: Figure S1. Genomic alignment computed with MAUVE between two of the sequenced poultry strains and the closest strain on the tree shown in Fig. 2: (A) PA18/15564 aligned to the DG5616 strain, (B) PA17/10312 aligned to the INSRALV892 strain.

Additional file 3: Figure S2. A) Tree of the $52 \mathrm{M}$. morganii strains analyzed in this study derived by alignment of the core genome using the program parsnp. The KT strain was used as a reference for the multiple genome alignment. B) The same tree but corrected for recombination. The red arrows indicate the cluster that shows differences between the two trees.

Additional file 4: Figure S3. Multiple alignment of an internal region of the RtxA toxin in the five poultry strains showing high sequence divergence.

Additional file 5: Table S2. List of candidate genes in the PA17/10312 strain with evidence for horizontal gene transfer from closely-related Morganellaceae species.

Additional file 6: Table S3. Annotation of active prophages in the five sequenced poultry strains.

Additional file 7: Table S4. Annotation of pathogenicity islands in the five sequenced poultry strains. Predicted genomic islands are coloured within the images based on the legend (generated through the web interface of IslandViewer 4 [50]). The internal line plot shows the similarity to the KT strain, taken as a reference.

Additional file 8: Figure S4. Genomic visualization of pathogenicity islands in the five sequenced poultry strains, performed in this study.

Additional file 9: Figure S5. Metabolic network of M. morganii strain PA17/10312 (computed through the web interface of BlastKOALA [31] in this study) showing two novel pathways specific to this strain: (A) Sucrose catabolism, sucrose $=>$ glucose, (B) Betaine biosynthesis, betaine $=>$ choline.

Additional file 10: Table S5. List of $M$. morganii strains used for the phylogenetic analysis with their respective NCBI accession IDs.

\begin{abstract}
Abbreviations
Al: Alien Index; AmpC: AmpC beta-lactamases; BLAST: The Basic Local Alignment Search Tool; COS: Columbia agar; ESBL: Extended-spectrum betalactamases; EUCAST: The European Committee on Antimicrobial

Susceptibility Testing; HGT: Horizontal Gene Transfer; MALDI-TOF MS: Matrix Assisted Laser Desorption/lonization-Time of Flight Mass Spectrometry; NGS: Next Generation Sequencing; SCS: Schaedler agar; SGC2: Sabouraud Gentamicin Chloramphenicol agar; SNPs: Single nucleotide polymorphisms
\end{abstract}

\section{Acknowledgements}

We thank Delfina Jandreski-Cvetkovics for her excellent technical assistance.

\section{Authors' contributions}

MA and MH conceived and designed the study. $\mathrm{CH}$ performed necropsy and bacteria isolation. MA performed the laboratory analyses. NP performed the bioinformatics analysis. MA and NP drafted the first version of the manuscript. All authors critically evaluated and interpreted the results. All authors provided feedback on the manuscript, read and approved the final version.

\section{Funding}

Not applicable.

\section{Availability of data and materials}

The datasets generated and/or analyzed during the current study are available in the $\mathrm{NCBI}$ repository (Accession IDs of the five sequenced poultry strains: WCIS00000000, WCIT00000000, WCIU00000000, WCIV00000000, WCIW00000000). The list of M. morganii strains used for the phylogenetic analysis and their respective NCBI accession IDs are listed in Supplementary Table 5.

\section{Ethics approval and consent to participate}

Sampling was performed during post-mortem investigations and complies with national legislation (Tierversuchsgesetz - TVG 2012, §1). Furthermore, the study was performed in co-operation with veterinarians in charge of the respective farms who have agreements with farm owners for applying veterinary procedures

\section{Consent for publication}

Not applicable.

\section{Competing interests}

The authors declare that they have no competing interests.

Received: 4 April 2020 Accepted: 17 August 2020

Published online: 24 August 2020

\section{References}

1. O'Hara CM, Brenner FW, Miller JM. Classification, identification, and clinical significance of Proteus, Providencia, and Morganella. Clin Microbiol Rev. 2000;13:534-46.

2. Adeolu M, Alnajar S, Naushad S, Gupta RS. Genome-based phylogeny and taxonomy of the 'Enterobacteriales': Proposal for enterobacterales ord. nov. divided into the families Enterobacteriaceae, Erwiniaceae fam. nov., Pectobacteriaceae fam. nov., Yersiniaceae fam. nov., Hafniaceae fam. nov. ,Morganellaceae fam. nov., and Budviciaceae fam. nov. Int J Syst Evol Microbiol. 2016;66:5575-99.

3. Liu H, Zhu J, Hu Q, Rao X. Morganella morganii, a non-negligent opportunistic pathogen. Int J Infect Dis. 2016;50:10-7.

4. Habila Mamman P, Kazeem HM, Raji MA, Nok AJ, Kwada J, Kwaga P, et al. Int Invent J Med Med Sci. 2014

5. Zhao C, Tang N, Wu Y, Zhang Y, Wu Z, Li W, et al. First reported fatal Morganella morganii infections in chickens. Vet Microbiol. 2012.

6. Kilonzo-Nthenge A, Rotich E, Nahashon SN. Evaluation of drug-resistant Enterobacteriaceae in retail poultry and beef. Poult Sci. 2013;92:1098-107.

7. Jones-Dias D, Clemente L, Moura IB, Sampaio DA, Albuquerque T, Vieira L, et al. Draft genomic analysis of an avian multidrug resistant morganella morganii isolate carrying qnrD1. Front Microbiol. 2016;7.

8. Chen YT, Peng HL, Shia WC, Hsu FR, Ken CF, Tsao YM, et al. Whole-genome sequencing and identification of Morganella morganii KT pathogenicityrelated genes. BMC Genomics. 2012;13 Suppl 7.

9. Olaitan AO, Diene SM, Gupta SK, Adler A, Assous MV, Rolain JM. Genome analysis of NDM-1 producing Morganella morganii clinical isolate. Expert Rev Anti Infect Ther. 2014;12:1297-305.

10. Minnullina L, Pudova D, Shagimardanova E, Shigapova L, Sharipova M, Mardanova A. Comparative genome analysis of Uropathogenic Morganella morganii strains. Front Cell Infect Microbiol. 2019;9:167. https://doi.org/10. 3389/fcimb.2019.00167.

11. Ishii S, Meyer KP, Sadowsky MJ. Relationship between phylogenetic groups, genotypic clusters, and virulence gene profiles of Escherichia coli strains from diverse human and animal sources. Appl Environ Microbiol. 2007;73: 5703-10.

12. Lawson B, Franklinos LHV, Rodriguez-Ramos Fernandez J, Wend-Hansen C, Nair S, Macgregor SK, et al. Salmonella Enteritidis ST183: emerging and endemic biotypes affecting western European hedgehogs (Erinaceus europaeus) and people in Great Britain. Sci Rep. 2018;8.

13. Galac MR, Lazzaro BP. Comparative genomics of bacteria in the genus Providencia isolated from wild Drosophila melanogaster. BMC Genomics. 2012;13.

14. Jacoby GA. AmpC B-Lactamases. Clin Microbiol Rev. 2009;22:161-82.

15. Bush LM, Calmon J, Johnson CC. Newer penicillins and beta-lactamase inhibitors. Infect Dis Clin North Am. 1995;9:653-86.

16. Carattoli A, Zankari E, Garciá-Fernández A, Larsen MV, Lund O, Villa L, et al. In Silico detection and typing of plasmids using plasmidfinder and plasmid multilocus sequence typing. Antimicrob Agents Chemother. 2014;58:3895903.

17. Parks DH, Imelfort M, Skennerton CT, Hugenholtz P, Tyson GW. CheckM: Assessing the quality of microbial genomes recovered from isolates, single cells, and metagenomes. Genome Res. 2015;25:1043-55. 
18. Jensen KT, Frederiksen W, Hickman-Brenner FW, Steigerwalt AG, Riddle CF, Brenner DJ. Recognition of Morganella Subspecies, with Proposal of Morganella morganii subsp. morganii subsp. nov. and Morganella morganii subsp. sibonii subsp. nov. Int J Syst Bacteriol. 1992;42:613-20.

19. Hedge J, Wilson DJ. Bacterial phylogenetic reconstruction from whole genomes is robust to recombination but demographic inference is not. MBio. 2014;5.

20. Minnullina L, Pudova D, Shagimardanova E, Shigapova L, Sharipova M, Mardanova A. Comparative genome analysis of uropathogenic morganella morganii strains. Front Cell Infect Microbiol. 2019. https://doi.org/10.3389/ fcimb.2019.00167.

21. Jones BD, Mobley HLT. Genetic and biochemical diversity of ureases of Proteus, Providencia, and Morganella species isolated from urinary tract infection. Infect Immun. 1987;55:2198-203.

22. Willis LM, Whitfield C. Structure, biosynthesis, and function of bacterial capsular polysaccharides synthesized by ABC transporter-dependent pathways. Carbohydr Res. 2013;378:35-44.

23. Rancurel C, Legrand L, Danchin EGJ. Alienness: rapid detection of candidate horizontal gene transfers across the tree of life. Genes (Basel). 2017.

24. Cosentino S, Voldby Larsen M, Møller Aarestrup F, Lund O. PathogenFinder Distinguishing Friend from Foe Using Bacterial Whole Genome Sequence Data. PLoS One. 2013;8.

25. Casjens S. Prophages and bacterial genomics: what have we learned so far? Mol Microbiol. 2003;49:277-300

26. Gillings MR. Integrons: past, present, and future. Microbiol Mol Biol Rev. 2014; 78:257-77.

27. Guo X, Rao Y, Guo L, Xu H, Lv T, Yu X, et al. Detection and genomic characterization of a morganella morganiiisolate from China that produces NDM-5. Front Microbiol. 2019

28. Mbelle N, Osei Sekyere J, Feldman C, Maningi NE, Modipane L, Essack SY. Genomic analysis of two drug-resistant clinical Morganella morganii strains isolated from UTI patients in Pretoria, South Africa. Lett Appl Microbiol. 2020;70: 21-8.

29. Mahrouki S, Bourouis A, Chihi H, Ouertani R, Ferjani MB, Moussa M, et al. First characterisation of plasmid-mediated quinolone resistance-qnrS1 coexpressed Bla CTX-M-15 and Bla DHA-1 genes in clinical strain of Morganella morganii recovered from a Tunisian intensive care unit. Indian J Med Microbiol. 2012;30:437-41.

30. Hacker J, Kaper JB. Pathogenicity Islands and the Evolution of Microbes. Annu Rev Microbiol. 2000:54:641-79.

31. Kanehisa M, Sato Y, Morishima K. BlastKOALA and GhostKOALA: KEGG Tools for Functional Characterization of Genome and Metagenome Sequences. J Mol Biol. 2016:428:726-31.

32. Wood JM, Bremer E, Csonka LN, Kraemer R, Poolman B, Van der Heide T, et al. Osmosensing and osmoregulatory compatible solute accumulation by bacteria. In: Comparative Biochemistry and Physiology - A Molecular and Integrative Physiology; 2001.

33. Wargo MJ. Homeostasis and catabolism of choline and glycine betaine: lessons from Pseudomonas aeruginosa. Appl Environ Microbiol. 2013;79: 2112-20.

34. Rahman A, Bhuiyan OF, Sadique A, Afroze T, Sarker M, Momen AMl, et al. Whole genome sequencing provides genomic insights into three Morganella morganii strains isolated from bovine rectal swabs in Dhaka, Bangladesh. FEMS Microbiol Lett. 2019;367.

35. Bradford PA. Extended-spectrum $\beta$-lactamases in the 21st century: characterization, epidemiology, and detection of this important resistance threat. Clin Microbiol Rev. 2001;14:933-51.

36. Servin AL. Pathogenesis of Afa/Dr diffusely adhering Escherichia coli. Clin Microbiol Rev. 2005;18:264-92.

37. Wurpel DJ, Beatson SA, Totsika M, Petty NK, Schembri MA. Chaperone-usher fimbriae of Escherichia coli. PLoS One. 2013;8:e52835. https://doi.org/10. 1371/journal.pone.0052835.

38. Spurbeck RR, Dinh PC, Walk ST, Stapleton AE, Hooton TM, Nolan LK, et al. Escherichia coli Isolates That Carry vat, fyuA, chuA, and yfcV Efficiently Colonize the Urinary Tract. Infect Immun. 2012;80:4115 LP-4122. https://doi. org/10.1128/IAl.00752-12.

39. Easton DM, Allsopp LP, Phan MD, Moriel DG, Goh GK, Beatson SA, et al. The intimin-like protein $\mathrm{FdeC}$ is regulated by $\mathrm{H}-\mathrm{NS}$ and temperature in enterohemorrhagic Escherichia coli. Appl Environ Microbiol. 2014;80:7337-47.

40. Bai $X$, Fu S, Zhang J, Fan $R, X u Y$, Sun $H$, et al. Identification and pathogenomic analysis of an Escherichia coli strain producing a novel Shiga toxin 2 subtype. Sci Rep. 2018;8:6756. https://doi.org/10.1038/s41598-01825233-x.

41. Andrade A, Pardo JP, Espinosa N, Perez-Hernandez G, Gonzalez-Pedrajo B. Enzymatic characterization of the enteropathogenic Escherichia coli type III secretion ATPase EscN. Arch Biochem Biophys. 2007;468:121-7.

42. Chatterjee A, Caballero-Franco C, Bakker D, Totten S, Jardim A. Pore-forming activity of the Escherichia coli type III secretion system protein EspD. J Biol Chem. 2015;290:25579-94.

43. Morgan JAW, Sergeant M, Ellis D, Ousley M, Jarrett $P$. Sequence analysis of insecticidal genes from Xenorhabdus nematophilus PMFI296. Appl Environ Microbiol. 2001:67:2062-9.

44. Alispahic M, Christensen H, Bisgaard M, Hess M, Hess C. MALDI-TOF mass spectrometry confirms difficulties in separating species of the Avibacterium genus. Avian Pathol. 2014;43:258-63.

45. Bauer AW, Kirby WM, Sherris JC, Turck M. Antibiotic susceptibility testing by a standardized single disk method. Am J Clin Pathol. 1966;493-6.

46. Seemann T. Prokka: rapid prokaryotic genome annotation. Bioinformatics. 2014:30:2068-9.

47. Orlek A, Phan $H$, Sheppard AE, Doumith M, Ellington M, Peto T, et al. A curated dataset of complete Enterobacteriaceae plasmids compiled from the NCBI nucleotide database. Data Br. 2017.

48. Song $W$, Sun $H X$, Zhang $C$, Cheng L, Peng $Y$, Deng Z, et al. Prophage hunter: an integrative hunting tool for active prophages. Nucleic Acids Res. 2019:47:W74-80.

49. Cury J, Jové T, Touchon M, Néron B, Rocha EP. Identification and analysis of integrons and cassette arrays in bacterial genomes. Nucleic Acids Res. 2016; 44:4539-50.

50. Bertelli C, Laird MR, Williams KP, Lau BY, Hoad G, Winsor GL, et al. IslandViewer 4: expanded prediction of genomic islands for larger-scale datasets. Nucleic Acids Res. 2017;45:W30-5.

51. Treangen T, Ondov B, Koren S, Phillippy A. Rapid Core-Genome Alignment and Visualization for Thousands of Intraspecific Microbial Genomes. bioRxiv. 2014:007351.

52. Lakin SM, Dean C, Noyes NR, Dettenwanger A, Ross AS, Doster E, et al. MEGARes: An antimicrobial resistance database for high throughput sequencing. Nucleic Acids Res. 2017;45:D574-80.

53. Steinegger $M$, Söding J. MMseqs2 enables sensitive protein sequence searching for the analysis of massive data sets. Nat Biotechnol. 2017;35: 1026-8.

54. Löytynoja A. Phylogeny-aware alignment with PRANK. Methods Mol Biol. 2014;1079:155-70.

55. Yang Z. PAML 4: phylogenetic analysis by maximum likelihood. Mol Biol Evol. 2007;24:1586-91.

56. Kanehisa M. The KEGG Database; 2003.

\section{Publisher's Note}

Springer Nature remains neutral with regard to jurisdictional claims in published maps and institutional affiliations.
Ready to submit your research? Choose BMC and benefit from:

- fast, convenient online submission

- thorough peer review by experienced researchers in your field

- rapid publication on acceptance

- support for research data, including large and complex data types

- gold Open Access which fosters wider collaboration and increased citations

- maximum visibility for your research: over $100 \mathrm{M}$ website views per year

At BMC, research is always in progress.

Learn more biomedcentral.com/submissions 